\title{
IMPLICATIONS OF A FUNDAMENTAL CONSCIOUSNESS
}

\author{
Copthorne Macdonald* \\ Charlottetown, Prince Edward Island, \\ C1A 8C5 Canada \\ Received June 22, 2010; accepted June 27, 2010
}

\begin{abstract}
The author postulates a universe in which mentality is as fundamental as physicality, explores the implications of that, and presents hypotheses concerning the evolution and functioning of human cognitive systems in such an environment. Of the several interpretations of reality which consider the physical and the mental to be equally real and equally fundamental, two compatible interpretations are discussed in detail. These are Ervin Laszlo's biperspectival system theory, and an elaboration of the perennial philosophy view which incorporates the concepts of algorithm, information, informational modulation, and evolution. In this latter view, reality comprises an enduring protophysical, protomental medium modulated by transient informational patterns. Chance-and-necessity evolution, guided by intrinsic "laws-of-nature" algorithms, produces systems that may be non-functional, physically functional, or physically and mentally functional. Given such a reality, the author concludes that human mentality evolved in bottom-up fashion, with mind-associated neuronal systems not so much creating mind as organizing a pre-existing propensity for awareness into useful, functional awareness, and providing for its modulation by useful information.

The author hypothesizes that discrete, localized occurrences of awareness accompany the functioning of relatively simple neuronal systems within the columnar structure of the brain's sensory cortex, and that each of these systems informationally modulates its associated awareness to create an elemental quale. Together, the awareness elements of a given sensory modality constitute a subjective field which, through brain activity not yet fully understood, becomes topologically related to the body image. The several superposed fields simulate a unified, multi-modal consciousness and constitute a global workspace. Selective attention to the qualia in these superposed fields activates neural correlates which facilitate situation evaluation and decision making.
\end{abstract}

Key words: Brain; Consciousness; Physics; Qualia

\section{INTRODUCTION}

As we assess progress on solving the "hard problem" in consciousness studies, certain contributions of David Chalmers and Bernard Baars seem particularly significant. In his recent writings, Chalmers has resurrected the call of earlier writers (e.g., Russell, 1921; Laszlo, 1972 a) to break with the physicalist view of reality. Chalmers (1995a) proposed "that conscious experience be considered a fundamental feature, irreducible to anything more basic," and suggested (1996) that perhaps some form of constrained double-aspect theory is appropriate. Baars' contribution (1988, 1997a, b) is a comprehensive "global workspace" model of cognitive functioning based on observed psychological and neurological information flows. It is a model which, in this author's view, is compatible with a fundamental mentality and with the hypotheses advanced here.

In this paper I postulate a universe in which mentality is as fundamental is physicality, explore the implications of that, and present hypotheses about the evolution and functioning of human cognitive systems in such an environment. Regarding the hard-problem element in Baars' theory, I address the dynamics of information transfer in both directions between physically-embodied and mentally-embodied information.

\section{PRIMAL REALITY: PHYSICAL-ONLY, OR MENTAL-PHYSICAL?}

One of the axioms of physicalist science is that the fundamental reality of the universe has only a physical na-

*Correspondence to: C. Macdonald, email: cop@copmacdonald.com 
ture - that reality has no mental or protomental qualities. We know that research in the highly-physical branches of science has flourished with this axiom in place. Doesn't this prove that it belongs there? Not necessarily. No problems arose, I would suggest, because in the fields of physics and chemistry the axiom was never really challenged. Where experiments involve overwhelmingly physical phenomena, it simply doesn't matter whether mentality exists or not. In those fields, the "everything is physical" assumption had little or no effect on the choice of experiments, or on the interpretation of experimental results. In trying to understand what the brain is up to, on the other hand, the assumptions made about primal reality matter a great deal. Most importantly, perhaps, they limit the range of hypotheses that are likely to occur to experimenters and theorists - or to be taken seriously by them if proposed by others.

For a long time there have also been interpretations of reality that see the physical and the mental as being equally real and equally fundamental. Forms of this perspective appeared first in the perennial philosophy traditions, later as dual-aspect monism and the biperspectival systems view, and most recently as panexperientialism. ${ }^{1}$ It is a way of looking at things that has been advocated for some time as a possible basis for solving the mind-body problem (Russell, 1921; Globus, 1976).

Globus (1973, p. 154) articulated the view this way: "mind is but one aspect of a fundamental neutral reality with matter being a second aspect, both aspects having equal importance, as two sides of a coin." Wald (1988, p. 9) used these words: "[Consciousness] is not some iffy phenomenon that we just project on reality; it is at the base, at the foundations." Wald also quoted Pauli:

"To us...the only acceptable point of view appears to be one that recognizes both sides of reality - the quantitative and the qualitative, the physical and the psychical - as compatible with each other, and can embrace them simultaneously. It would be most satisfactory if physis and psyche (i.e., matter and mind) could be seen as the complementary aspects of the same reality." (p. 11) Rensch (1971, p. 297) said:

"There is no contrast between mind and matter. We must recognize that all "matter" is protopsychical in character."

Like the statements above, most statements about psycho-physical reality tell us little about how fundamental consciousness might actually work. System theorist Ervin Laszlo's biperspectival systems theory (1972a) is more specific. Laszlo, too, considered mentality to be fundamental. But he also held that specific expressions of mentality occur in a systems context, and that each system experiences "sensations appropriate to the organization of the system" (1972a, p. 172). Just as Laszlo saw consciousness going all the way down, he also saw systems going all the way down. In the view he articulated, the universe is a hierarchy of "natural systems" with the systemic structures at one level in the hierarchy being components in the systems at the next, more complex level. His hierarchy included atoms, molecules, inorganic crystals, organic cells, multi-cellular systems within organisms, and whole organisms. Individual systems at all levels are "biperspectival". That is, each system can be experienced objectively from the outside as a physical entity. But each is also capable of experiencing aspects of its own functioning. Laszlo ascribed subjectivity to systems as simple as atoms and molecules, and speculated that an atom would experience major events such as an electron jumping from one orbit to another or the fission of its nucleus.

Laszlo also called attention to those much more complex systems which play a subjective role in cognition. He called these latter systems "natural-cognitive" systems, and said of them:

"[Natural-cognitive systems] are . . . 'biperspectival': They are single, self-consistent systems of events, observable from two points of view. When 'lived' such a system is a system of mind-events, viz., a 'cognitive system'. When looked at from any other viewpoint, the system is a system of physical events, i.e., a 'natural system'. The physical and mental sets of events are correlates ..." (1972a, p. 154)

In such a cognitive system there would be closed, inaccessible subjectivities within each component of the system - each atom, molecule, and cell — as well as the "mind-event" subjectivity associated with the system as a whole.

When we contemplate psycho-physical theories such as Laszlo's, it is important that we don't anthropomorphize. Although all physically eventful systems will also be mentally eventful, the mental events of most systems are not going to be anything like human mental events. Nor are they likely to be useful. This is obviously true of the physical utility of systems. If I take a handful of electronic parts at random, interconnect them in some unplanned way, and apply a voltage between two points, something electrical will probably happen. A current may flow; parts may heat. But nothing particularly useful is likely to happen. Only a tiny percentage of possible systemic structures and energizing arrangements allow interesting and useful physical happenings. We must expect the same to be true of the mental aspect of physical systems. Even if a system has been carefully designed for physical utility, there is no reason to suspect that its mental aspect will serve any sort of useful function. The analytical challenge is to find out what sort of physical arrangements give rise to useful mentality. Later in this paper I share some of my own analysis, and discuss what I consider to be the systemic roots of the human "global workspace" mental environment. 
Laszlo's theory gives us a rough sense of mental happenings at the whole-system level, but very few details. Fortunately, a recent elaboration of the perennial philosophy view - one that incorporates the concepts of algorithm, information, informational modulation, and evolution - allows us to see more. Laszlo's theory and this other view are based on similar assumptions about primal reality, and both focus on the role of systems. But this new perspective's kit of conceptual tools helps us to explore more deeply the why and how of psychophysical systems, and to better understand the processes going on within them.

The perennial philosophy perspective was first introduced to many English-speaking readers by Aldous Huxley (1945), but the view has long been espoused by Vedanta, Taoism, the Zen and Dzogchen branches of Buddhism, and Christian mysticism. It is "a view which holds that underlying the transient, ever-changing realm of manifest existence is an eternal unmanifested Ground, a protophysical, protomental oneness that interpenetrates existence and enables it to be." (Macdonald, 1994, p. 136)

The ancients were able to apprehend this perspective intuitively, but had difficulty expressing it clearly in words because they lacked certain key concepts that we now have. The present "information age" or postmodern refinement of the perspective had to wait until these concepts emerged and became part of our cultural vocabulary - concepts like medium and message, carrier and modulation, algorithm and information. It had to wait, for instance, until Claude Shannon (1948) demonstrated that the fuzzy concept of information could have a solid, quantifiable meaning. It had to wait until DNA research in the 1950s showed that organisms and other physical structures are in fact informational structures. It had to wait until the role of algorithms in information processing became clear in the 1960s and 1970s, and until ideas about parallel processing began to emerge in the 1980s. In essence, the interpretation is this:

Reality consists of an enduring medium modulated by transient informational patterns.

- The medium has a physical aspect, a mental aspect, and an algorithmic aspect.

- Physically, the primal medium is energy or protoenergy - the ground of the physical universe.

- Mentally, the primal medium is awareness or protoawareness - the ground of mind and subjective experience.

- Algorithmically, the medium is the home of "laws of nature" algorithms which, during the life of the universe, allow mental/physical potentials to become actualized. These algorithms, these laws, are the intrinsic rules that guide physical, chemical, geological, and bio- logical change. It is the moment-to-moment functioning of these algorithms that form patterns, and modulates the primal medium with information - creating, as it does, that hierarchy of systems we call the universe. In information processing terms, the universe is equipped with built-in recursive algorithms which repeatedly take the informational situation that exists at this instant and transform it into a new informational situation in the next. (Macdonald, 1994, p. 136)

At $t=0$, the instant of the Big Bang, our universe is generally conceived as just the medium with no, or almost no, informational patterning. Within a small fraction of a second after $t=0$, however, the informational patterning or modulating began (Barrow \& Silk, 1980). This information-creating, system-building process has been described for the early universe by Weinberg (1988), for the later universe by Laszlo (1972a, 1972b, 1987), and overall in narrative form by Swimme and Berry (1992). A hierarchy of natural systems evolved in response to the guidance offered by those intrinsic algorithms.

In this view, the physical universe is seen to be an informational patterning of energy. At the submicroscopic level that patterning appears as varieties of energy/matter quanta, and as atomic and molecular structure. At micro-, meso-, and macroscopic levels it appears as the space-time patterning of those components in complex geological, biological, and astronomical systems. The enduring reality is the energy itself (it cannot be created or destroyed), but what makes the universe so interesting is the ever-changing informational patterning which energy undergoes as evolution proceeds.

\section{INFORMATION, AWARENESS, QUALIA, MIND, AND ATTENTION}

The essence of information is difference, or as Gregory Bateson put it: "any difference which makes a difference" (1975, p. 381). Plato may have imagined disembodied information, perfect patterns existing all by themselves on some ethereal plane. But real-world information is not like that; differences that make a difference must be differences in something. Information must have its medium or carrier. So information isn't a thing-in-itself, it is a pattern of significant differences impressed upon some substrate or medium that serves as its carrier. There are both encoded information and embodied information. Examples of encoded information include written language, DNA, and the ones and zeros of computer data. Examples of embodied information include the shape of an ocean wave, the structural and functional organization of a living organism, and the details of a conscious experience. Viewing physical 
existence as informationally-patterned energy meets little resistance these days. Mathematician Rudy Rucker has said: "It is now considered reasonable to say that, at the deepest, most fundamental level, our world is made of information. . . . For postmodern people, reality is a pattern of information, a pattern in fact space" (1987, p. 31). Physicist John Wheeler put it this way: "all things physical are information-theoretic in origin" (1994, p. 296), and added, "Tomorrow we will have learned to understand and express all physics in the language of information" (1994, p. 298).

In addition to being a carrier of physical information, a psycho-physical primal reality is also a carrier of mental information. We have labels for its mental-carrier aspect: awareness, sentience, pure subjectivity, consciousness without content. ${ }^{2}$ I use the term awareness because there are few one-word alternatives, and because much of the perennial philosophy literature uses it. ${ }^{3}$ Beyond labeling this carrier, we can refer to its roles and functions: Awareness and energy are the two faces of the primal reality, and in the same sense that energy is the enabler of physical activity, awareness is the enabler of mental activity. Awareness is the ground, medium, and carrier of mind. Awareness is what permits conscious experience. Awareness is the subjective capability that is present during ARAS (ascending reticular activating system) arousal. Awareness is the sentient medium that when modulated by neuronallygenerated information becomes mental qualia, mind with informational content. But because awareness is primal and unique, our powers of definition are limited. As with other one-of-a-kind aspects of primal reality (energy, time, space), a detailed analogic definition of awareness is not possible.

Traditionally, definitions of primal entities have left much to be desired. Asked to define time, Einstein is supposed to have said: "Time is what clocks measure." A widely-accepted definition of energy is: "Energy is the capacity to do work." About awareness we might say: "Awareness is the capacity to present information subjectively." Each definition is saying something interesting about the primal entity it attempts to define, but each falls short of letting us look at the intrinsic nature of that entity. To me, this difficulty in defining awareness is itself strong evidence that this aspect of reality which I'm labeling awareness - like those other aspects: energy, time and space - is fundamental, not derivative.

In the view presented here, awareness and its informational modulations are intimately associated. This intimacy is analogous to the intimacy between the ocean and its wave modulations or a radio station's energy carrier and its program modulations. In this view there is no observer/observed duality, no "ghost in the machine." There is no experiencer separate from experience; there is just experiencing. The casual observer of mind does see a subject/object duality, but adepts in advanced perennial philosophy traditions such as Zen and Dzogchen have seen through the apparent duality and have become cognizant of the inherently non-dual nature of mental experience. ${ }^{4}$ Varela also commented on this:

"One of the originalities of the phenomenal attitude is that it does not seek to oppose the subjective to the objective, but to move beyond the split into their fundamental correlation." (1996, p. 339)

Those accomplished meditators and Western phenomenologists who have paid the price in time and effort to, in Wilber's words, "become adequate to the postformal data" represent a "validating community of competent subjects" who attest to this. ${ }^{5}$

Before proceeding, I'd like to clarify my use of three other terms. Qualia are awareness-associated informational artifacts. They are components of the informational totality which constitutes conscious experience. They are specific instances of informationallymodulated awareness. They are mind content. Edelman has said: "Qualia constitute the collection of personal or subjective experiences, feelings, and sensations that accompany awareness. They are phenomenal states "how things seem to us" as human beings. For example, the "redness" of a red object is a quale. Qualia are discriminable parts of a mental scene that nonetheless has an overall unity. They may range in intensity and clarity from "raw feels" to highly refined discriminada." (1992, p. 114)

In another sense, qualia are mental metaphors that symbolize the modulating stimulus, or are elements of a mental analog of some perceived reality (Jaynes, 1982). For example, specific odors are mental metaphors for specific air-borne molecules. And visual experience is a reasonably accurate mental analog (map) of the optical scene projected on the retinas.

Mind is the space-like awareness environment in which qualia appear, the subjective global workspace, the composite experiential field. Attention is a condition of mind and brain marked by heightened, and usually selective, noting of qualia. Research tells us that selective attention is not caused by varying the intensity of awareness - which is simply present during periods of arousal and not present during deep sleep - but by selectively gating neuronal information. ${ }^{6}$ The usual human pattern is to attend intently to one quale or aspect of a quale at a time, although wide-focus, multi-qualia modes of attending can be learned (Fehmi and Fritz, 1980).

\section{WHY EVOLVE A MIND?}

I agree with Chalmers when he said, "For a satisfactory theory, we need to know more than which processes give rise to experience; we also need an account of why and how. A full theory of consciousness must build an 
explanatory bridge" (1995b). The model of conscious functioning that my own mind-watching and literaturereview activities have led me to adopt is a theater model, similar to, and I believe largely compatible with, Bernard Baars Global Workplace Theory (Baars, 1988, 1997a, b; Newman and Baars, 1993). What I propose to contribute to that view is some insight into why and how the global workplace came into existence, and perhaps a few fresh thoughts about its functioning.

Let's begin with the key question posed in Vol. 1, No. 2 of the Journal of Consciousness Studies: "Why, in principle, should a neuronal system of any degree of complexity give rise to the phenomenal experience of consciousness?" My answer has two parts: If we start with radical physicalist assumptions about the nature of reality, then there is no reason why, "in principle". a neuronal system should give rise to conscious experience. If, however, we start with the assumption that primal reality is as described in this paper, then the arising of conscious experience is not astonishing at all. It's just what we might expect. If reality is this second way, then the role of the neuronal system is not to mysteriously create awareness and mind from alien substance. Rather, it is to organize a pre-existing propensity for awareness into useful, functional awareness, and provide for its modulation by useful information. Again we face the utility issue. If awareness is as primal and ubiquitous as energy, then it will be present in every system. But whether or not it plays a functional role will depend on how a particular system has been configured, and the nature of its connections to the world outside it.

It is quite possible that during pre-biological evolution (the cosmological and geological phases of evolution) awareness played no functional role. It is clear, however, that at some point during biological evolution, awareness was harnessed and put to work. If, today, we humans were given the task of designing systems that have useful mental characteristics, we quite literally would not know where to begin. Yet evolution - with its slow, plodding, and chance-and-necessity geniusdid a magnificent job of it. As I see it, this was possible because the medium on which the cosmic algorithms have been operating is a mental-physical medium, not just a physical one.

Early in the evolution of living things, organisms exhibited sensitivity to their environments, and some were able to respond to environmental changes. Many types of plants align themselves to maximize their exposure to light. Paramecia move away from irritating stimuli. These behaviors appear to most scientists to arise from totally physical, reflex-like mechanisms. They feel that subjectivity and mind play no functional role, and this may well be true. It may even be true of amphibians. In the classic Lettvin, J. et al. (1965) study of "What the frog's eye tells the frog's brain". the frog's eye is reported to have different types of retinal sensors that give rise, quite automatically it seems, to a limited set of stereotyped behaviors. If a small dark object passes across the frog's field of vision (a fly, perhaps?), the frog's tongue reflexively darts out. If the overall light level suddenly drops (the shadow of a hawk, perhaps?) the frog reflexively jumps off the lily pad. Whether or not these organisms have a functional consciousness may become clear when we finally understand how human mentality works at the neural level.

As evolution progressed, species proliferated and ecosystems became more complex. Increasingly intricate forms of animal life emerged to take advantage of newly created environmental niches. Increasingly sophisticated sensory systems evolved. New survival opportunities opened up; but so did new threats to survival. As a result of all this, evolutionary pressure arose for better situation-analysis and decision-making capabilities.

With just one perceptual mode (say chemical sensitivity or taste) and a limited number of action modes (say eating and flight), purely physical, reflex-type control of behavior would appear to be adequate. But in organisms equipped to receive several different kinds of highlydetailed sensory information simultaneously, and to respond in many different ways, the demands on the control system increase dramatically. To avoid behavioral chaos or gridlock from competing information, sophisticated analysis and decision-making systems were needed, and eventually evolved.

Artificial intelligence research has shown that it is not easy to sort out the relevant from the irrelevant, or even to make figure/ground discriminations using physicalonly approaches (Hurlbert and Poggio, 1986). I am suggesting that mentality - and selective attention in particular - was evolution's way of solving this problem. I am not saying that it is impossible, in principle, to perform these same tasks using only the physical aspect of systems and computational techniques. Rather, I'm hypothesizing that this is not what evolution did. If we human designers were given the task, we would be forced to take an all-physical approach (the only one with which we are currently familiar), and given sufficient time and resources we might eventually succeed in solving the problem that way. Evolution, however, had another design option, one not yet open to us: the option of incorporating mentality as well as physicality into its designs. This, of course, is not a new idea. Baars (1997a) notes William James's comment: "The study . . . of the distribution of consciousness shows it to be exactly such as we might expect in an organ added for the sake of steering a nervous system grown too compli- 
cated to regulate itself" (James, 1890/1983, p. 141). Theorist of evolution Harry Jerison put it this way: "I regard the mind and conscious experience as constructions of nervous systems to handle the overwhelming amount of information that they process" (1973, p. 4).

Evolution's mental-physical approach seems to have been this: Create neuronal systems that generate mental metaphors or analogs of the immediate physical situation, and bring them together in one mental "space." Combine this with a selective attention mechanism which allows the superposed mental fields to be scanned for qualia that have survival or reproductive significance, and arrange for the neuronal correlates of attended-to qualia to become available for unconscious computational processing. ${ }^{7}$

In humans, if the processing deems the quale to be extremely significant it will occasionally give rise to an automatic reaction, as when someone jumps immediately to save a child in danger. Usually, however, the computational processing simply suggests a course of action. It does this by causing a thought, feeling, or emotion to appear in the mind along with the information already present there. A third possibility arises when the processing decides that the quale being attended to is not significant. In that case, an unconscious instruction restarts attentional scanning.

In this view, the conscious field is the great simplifier. The creation of a mental "workspace" allows large amounts of relevant and irrelevant information to be brought together in one subjective arena. Selective attention then allows that mass of data to be rapidly surveyed in serial fashion. Neuronal correlates of items attended to are checked computationally for relevance. Different kinds of relevance cause different kinds of data-processing outputs to appear in the mind: mental images; thoughts; and feelings of pleasure, pain, fear, anger, hate, or tenderness, for example. These newlyarisen qualia are themselves then available for possible selection by attention, and if selected, their neuronal correlates would be used as input data for further processing. At the end of all the processing, the ultimate behavioral decision is frequently accompanied by a mental correlate of its own - a YES feeling, a NO feeling, fear, anger, etc.

This kind of analysis-and-control system is flexible. Because evolution's behavioral suggestions - lust, fear, hunger, anger, a liking for sugar, etc. - are presented mentally, they are not hard-wired action imperatives. Priorities can be accommodated; "Flee the tiger rather than grab the fruit". And learning can override evolutionary messages; "I'm already overweight, so I'll ignore the impulse to have dessert." Through our immersion in culture we acquire a learned hierarchy of values which can, in various circumstances, override evolution's default hierarchy. If our learning has been appropriate to our reality, then we will behave appropriately and wise- ly in a much wider range of circumstances than if our control system had been hard wired in another era.

\section{SOME NEUROLOGICAL SPECULATIONS}

Postulating that we live in a two-aspect, biperspectival universe allows us to take seriously the possibility that for a relatively primitive mentality to come into existence, an extremely complex brain is not needed. If subjectivity is fundamental, then we should expect that relatively simple forms of mind would require only relatively simple systems for their arising. It appears that even in the human brain, awareness may not arise as one unified whole, but through the integration of many localized instances of awareness, each of which is associated with the functioning of a relatively simple local system.

Although human awareness seems unified, research during the past few decades indicates that it is not. Hebb (1980, p. 40), in referring to the split-brain patients of Bogen, Gazzaniga and Sperry, notes that after the two brain hemispheres were separated surgically "a patient might seem to have two minds, a left-hand and a righthand mind." MacLean (1977, p. 313) saw "three mentalities." He felt that each part of the "triune brain" (reptilian brain, limbic system, and neocortex) has "its own subjectivity." Jackendoff (1990, p. 52) saw yet another "disunity in awareness" - a disunity in which each sensory modality has its own separate awareness.

I am suggesting that the separate subjective fields associated with vision, somatosensory experience, hearing, smell, and taste are each made to relate topologically to the body image by processes of arrangement and binding not yet fully understood. The effect is a superpositioning of the individual fields. They overlay and overlap much like multiple transparencies laid on an overhead projector, with all five sensory fields occupying roughly the same subjective space. ${ }^{8}$ Together, the superposed fields simulate a unified multi-modal consciousness, and constitute a subjective global workspace.

I am also suggesting that even within a single brain region or sensory modality, awareness may not be unified. Let us think for a moment about the discrete nature of the information involved in visual perception. Although human visual experience seems smooth and continuous, we know that the information which gives rise to it is actually discrete, segmented, elemental. Being derived from the outputs of discrete retinal neurons, visual experience is like a computer image or newspaper picture in that it contains a limited number of visual elements. Visual experience seems continuous because the elements are tightly packed, and because there are so many of them. But it is not continuous. The seemingly smooth and continuous experiences of sound and touch also originate in impulses from discrete sensory neurons in the cochlea and skin. If the information that fills the 
various sensory fields seems continuous but isn't, why assume that the awareness associated with that information is continuous? I am suggesting that it is more reasonable from an evolutionary and systems point of view to assume that each element of conscious data is associated with its own element of awareness.

My hypothesis is that in the visual, auditory, and somatosensory cortex of the human brain there exist a multitude of relatively simple neuronal systems, each of which organizes the medium's inherent subjective capacity into a functioning elemental awareness. If an appropriate neuronal signal is received from an external source, each of these mind-element systems also informationally modulates its elemental awareness, creating in the process an elemental quale - a pictorial element in the case of visual perception, part of an auditory experience, or a localized sensation in the somatic sensory field. The binding and orienting problems still need to be solved, but it may turn out that what is being bound and oriented are a large number of quite small elements of awareness - each with its associated informational modulation.

The visual system has been more extensively studied than the auditory or somatosensory systems, and to support "the involvement of early parts of visual cortex in visual consciousness." Baars (1997a) presented four "convergent lines of evidence" (p. 68):

First, when Area V1 is lost, people report a loss of visual conscious experience, though they can still "guess" at the objects their eyes are looking at. Second, when the early visual areas are stimulated by a gentle current, people report conscious visual flashes (phosphenes). Third, when people are conscious of a visual object, we can see the early visual areas "light up" in PET scans, indicating a distinct increase in neural activity. Fourth, recording of single cells in visual cortex indicates that there is a difference between the conscious and unconscious flows of stimulation (pp. 100-01).

Cortical areas A1 and S1 play roles in auditory and somatosensory perception analogous to $\mathrm{V} 1$ 's role in vision. The bilateral destruction of A1 produces cortical deafness (Aitkin, 1990), and the destruction of S1 results in the inability to consciously perceive stimuli at the body surface (Schmidt, 1986). Thus, it seems likely that $\mathrm{A} 1$ and $\mathrm{S} 1$ are where the space-like fields of auditory and somatosensory awareness and qualia are generated - although secondary processing areas might also be involved. The neural columns which are present in these sensory processing areas seem likely candidates for those awareness-organizing, awareness-modulating subsystems mentioned above. One suggestive piece of data is that the observed resolution of visual data in V1 is similar to column spacing. F.T. Hambrecht (1992) electrically stimulated the foveal region of V1 and determined that phosphenes produced by simultaneous stimulation through two electrodes 500 microns apart appeared as two separate phosphenes, but as just a single phosphene when electrodes were 250 microns apart. Another reason for highlighting these neural columns is that cortical levels 2, 3, and 4 appear to be involved in the processing of sensory data, and levels 5 and 6 appear to be involved in arousal and the presence or absence of consciousness. ${ }^{9}$ This suggests the possibility that neurons in layers 5 and 6 may be involved in organizing awareness tendencies into useful awareness, and that neurons in the middle layers may be involved in modulating that awareness with sensory data and "sensory-like" imagined or remembered data.

It is also possible to describe the situation from Laszlo's "biperspectival system" point of view. From that perspective, those small awareness-organizing neuronal systems are subsystems of the overall "global workspace" cognitive system, and their functioning is an integral part of the larger system's functioning. As modulating signals from the computational environment are processed by the columnar subsystems, subsystem physical states constantly change - elemental physical events occur. Those physical changes are accompanied by correlated changes in subsystem subjective states elemental mental events occur.

The many mind-element subsystems, together with those brain subsystems responsible for their topological organization, constitute a single "natural-cognitive system" whose interior state is the subjective global workspace. Human consciousness is this system's selfexperience.

\section{NEXT STEPS}

What's next? What can be done to investigate bottomup hypotheses such as those put forth here? How can we test their validity and determine their worth? A few thoughts:

- First, previous experimental work could be reconsidered with the present set of ideas in mind. Would this, in at least a few situations, lead to a different interpretation of the original data? Is there previous experimental work that clearly refutes the speculations and hypotheses put forth in this paper? Is there work that lends support or confirmation?

- Could an experiment be devised to temporarily block arousal in a portion of V1 without interfering with information processing activities in the blocked region? How would this affect subjective experience? And what would the change in subjective experience tell us about the validity of the ideas presented here? Obviously, it is important to continue work on the binding problem, and 
to search for the mechanism which aligns whole fields of awareness elements with the body image. Equally obvious is the importance of lesion analysis and brainscan technology in shedding light on brain/mind correlations. The eventual task will be to "reverse-engineer the wetware" to determine, in detail, how neural functioning actually does give rise to subjective experience. But first we need to know that we are heading in the right direction. We need experiments that help determine whether or not consciousness really is a bottom-up phenomenon, a fundamental feature of the universe.

\section{NOTES}

1 For a discussion of panexperientialism see de Quincey (1994).

2 Can we really talk about consciousness without content, awareness without informational modulation, mind without qualia? Absence of mind content is not part of our everyday experience, but accomplished meditators approach this condition. They find that during prolonged periods of meditation, mind content drops toward the asymptotic limit of no content. They also find that awareness remains present, irrespective of the level of mental information. At some point in their practice, many advanced practitioners experience a cognitive shift in which awareness becomes figure rather than ground, and is thereafter recognized as a tangible reality in its own right.

3 I do realize that the term is also used in other senses - Chalmers" use, for example (1996, p. 28).

4 Varela has suggested that both Western Phenomenology (1996) and Buddhist meditation (Varela, et al., 1991) are useful techniques for the serious investigation of conscious experience. Wilber $(1997 \mathrm{a}, \mathrm{b})$ and I (Macdonald, 1996) have made similar suggestions. Disciplines that I have found particularly fruitful are the Vipassana and Dzogchen varieties of Buddhist meditation, and the Western practice called Open Focus (Fehmi and Fritz, 1980).

5 Where appropriate, researchers will have to engage in various injunctions that transform their own consciousness if they are to be adequate to the postformal data. You cannot vote on the truth of the Pythagorean Theorem if you do not learn geometry (the injunction); likewise, you cannot vote on the truth of Buddha Nature if you do not learn meditation. All valid knowledge has injunction, apprehension and confirmation; the injunctions are all of the form, "if you want to know this, you must do this" - and thus, when it comes to consciousness studies itself, the utterly obvious but much-resisted conclusion is that certain interior injunctions have to be followed by the researchers themselves. If we do not do this, then we will not know this (1997a, p. 91).

6 Newman and Baars (1993) report that "virtually all information conveyed to the cortex from visual, auditory, and somatosensory pathways is relayed through the thalamus" and that "gatelets" in the reticular nucleus of the thalamus regulate the "flow of information from the thalamus to the cortex." They report that these gatelets are opened and closed in response to control signals from various regions of the cortex.

7 A number of researchers have presented results that link conscious experience and the attentional process to specific physical brain correlates. For example, Wurtz, Goldberg, and Robinson, reporting on their primate research, said: If the monkey is alert, but not attending to anything in particular, the response of the nerve cells in [the posterior parietal cortex] is relatively uniform. When the monkey begins to attend to some object, however, the nerve cells in the posterior parietal cortex that are related to the object because it is in their receptive field begin to discharge more intensely. . . . We do not know how the enhancement arises, and in the absence of this knowledge it is tempting to say that the enhanced response amounts in itself to visual attention. It remains quite possible, however, that the enhancement is only a correlate of visual attention (1982, p. 124).

It is also possible to pay attention to visual attributes other than position, and to detect, in the secondary visual processing areas, physical correlates of that kind of attending. As Corbetta, Miezin, Dobmeyer, Shulman, and Petersen found: "Attention to basic visual attributes such as shape, color, or velocity appears to influence ... physiological measures of visual processing. . . . Physiologically, neural activity is increased in extrastriate regions specialized for processing information related to the selected visual attribute" (1990, p. 1556).

8 Should you wish to investigate this for yourself, I have elsewhere (Macdonald 1993) described a little experiment: "If I look straight ahead and move my hands around to find the edges of the visually-active part of the field, I conclude that it's about one unit high and three or four wide. If I then put a bottle of perfume under my nose, the experience of odour fills much of this same field - being most intense in the centre, less so at the edges. If I next bite into a piece of fruit and pay attention to my experience of taste as I chew it, I find that taste occupies a more sharply defined zone than odour. Taste sensations appear in a horizontallyoriented oval located below the centre of visual data. Touch, body sensations, and sounds also appear in the awareness field, sometimes extending beyond the edges of the visual data. The location of most body feelings and sensations is sharply defined, while the direction of most sounds is just roughly indicated" (pp. 25-6).

9 See, for example, Livingstone and Hubel (1981).

\section{REFERENCES}

Aitkin, L. (1990). The Auditory Cortex: Structural and functional bases of auditory perception. London: Chapman and Hall. 
Baars, B.J. (1988). A cognitive theory of consciousness. Cambridge: Cambridge University Press.

Baars, B.J. (1997a). In the Theater of Consciousness: The Workplace of the Mind. New York: Oxford University Press.

Baars, B.J. (1997b). In the Theatre of Consciousness: Global Workspace Theory, A Rigorous Scientific Theory of Consciousness. Journal of Consciousness Studies, 4, 292-309.

Barrow, J. D., \& Silk, J. (1980). The Structure of the Early Universe. Scientific American, 242, 118-128.

Bateson, G. (1975). Steps to an Ecology of Mind. New York: Ballantine Books.

Chalmers, D.J. (1995a). The puzzle of conscious experience, Scientific American, December, 80-86.

Chalmers, D. J. (1995b). Facing up to the problem of consciousness. Journal of Consciousness Studies, 2, 200-19.

Chalmers, D.J. (1996). The Conscious Mind: In Search of a Fundamental Theory. New York: Oxford University Press. Corbetta, M., Miezin, F. M., Dobmeyer, S., Shulman, G.L., \& Petersen, S. E. (1990). Attentional Modulation of Neuronal Processing of Shape, Color, and Velocity in Humans. Science, 248, 1556-1559.

de Quincey, C (1994). Consciousness All the Way Down? An Analysis of McGinn's Critique of Panexperientialism. Journal of Consciousness Studies, 1, 217-229.

Edelman, G.M. (1992). Bright Air, Brilliant Fire: On the Matter of the Mind. New York: Basic Books.

Fehmi, L. G. \& Fritz, G. (1980). Open Focus: The Attentional Foundation of Health and Well-Being. Somatics, 2, 4-30.

Globus, G. (1973). Consciousness and Brain I, The Identity Thesis. Archives of General Psychiatry, 8, 153-160.

Globus, G. (1976). Mind Structure and Contradiction. In G. Globus, G. Maxwell, \& I. Savodnik, (Eds.). Consciousness and the Brain: A Scientific and Philosophical Inquiry. New York: Plenum Press.

Hambrecht, F. T. et al. (1992). Feasibility of a Visual Prosthesis for the Blind Utilizing Intracortical Microstimulation. (Author-paginated reprint), Proceedings of the 4th Vienna International Workshop on Functional Electrostimulation, Baden, Austria: Department of Biomedical Engineering and Physics, University of Vienna.

Hebb, D.O. (1980). Essay on Mind. Hillsdale, NJ: Lawrence Erlbaum Associates.

Humphrey, N. (1992). A History of the Mind: Evolution and the Birth of Consciousness. New York: Simon and Schuster.

Hurlbert, A. \& Poggio, T. (1986). Do Computers Need Attention? Nature, 321,651 .

Huxley, A. (1945). The Perennial Philosophy. New York and London: Harper and Brothers.

Jackendoff, R. (1990), Consciousness and the Computational Mind. Cambridge, MA: MIT Press.

James, W. (1890/1983), The Principles of Psychology. Cambridge, MA: Harvard University Press.

Jaynes, J. (1982). The Origin of Consciousness in the Breakdown of the Bicameral Mind. Boston: Houghton-Mifflin.

Jerison, H. J. (1973). Evolution of the Brain and Intelligence. New York: Academic Press.

Laszlo, E. (1972a). Introduction to Systems Philosophy: Toward a New Paradigm of Contemporary Thought. New York: Gordon and Breach.
Laszlo, E. (1972b). The Systems View of the World. New York: George Braziller.

Laszlo, E. (1987). Evolution: The Grand Synthesis. Boston: Shambhala.

Lettvin, J. et al (1965). What the frog's eye tells the frog's brain. In W. McCullock, Ed., Embodiments of Mind. Cambridge, MA: MIT Press.

Livingstone, M. S. \& Hubel D. H. (1981). Effects of sleep and arousal on the processing of visual information in the cat. Nature, 291, 554-561.

Macdonald, C. (1993). Toward Wisdom. Toronto: Hounslow Press.

Macdonald, C. (1994). An Energy/Awareness/ Information Interpretation of Physical and Mental Reality', Zygon: Journal of Religion and Science, 29, 135-151.

Macdonald, C. (1996). The necessity for developing skill at mind-process observation, and a suggested methodology. Presented at the Tucson II Toward a Science of Consciousness 1996 conference.

MacLean, Paul (1977), On the Evolution of Three Mentalities. In S. Arieti and G. Chzanowki (Eds.), New Dimensions in Psychiatry: A World View, Vol. 2, chap. 15. New York: John Wiley and Sons.

Newman, J. \& Baars B. J. (1993). A Neural Attentional Model for Access to Consciousness: A Global Workspace Perspective. Concepts in Neuroscience, 4, 255-90.

Rensch, B. (1971). Biophilosophy. New York: Columbia University Press.

Rucker, R. (1987). Mind Tools: The Five Levels of Mathematical Reality. Boston: Houghton Mifflin Co.

Russell, B. (1921). The Analysis of Mind. London: George Allen and Unwin.

Schmidt, R. F. (Ed.) (1986). Fundamentals of Sensory Physiology. Berlin: Springer-Verlag.

Shannon, C.E. (1948). A mathematical theory of information. Bell System Technical Journal, 27, 379-423, and 623-656.

Swimme, B., \& Berry, T. (1992). The Universe Story. San Francisco: Harper San Francisco.

Varela, F.J., Thompson, E., \& Rosch, E. (1991). The Embodied Mind: Cognitive Science and the Human Experience. Cambridge, MA: MIT Press.

Varela, F.,J. (1996). Neruophenomenology: A methodological remedy for the hard problem. Journal of Consciousness Studies, 3, 330-49.

Wald, G. (1988). Cosmology of life and mind. Los Alamos Science: Fellows Colloquium, Issue 16.

Weinberg, S. (1988). The First Three Minutes: A Modern View of the Origin of the Universe. (Updated ed.) New York: Basic Books.

Wheeler, J.A. (1994). At Home in the Universe. Woodbury, NY: American Institute of Physics Press.

Wilber, K. (1997a). An Integral Theory of Consciousness. Journal of Consciousness Studies, 4, 71-92.

Wilber, K. (1997b), The Eye of Spirit: An Integral Vision for a World Gone Slightly Mad. Boston: Shambhala.

Wurtz, R. W., Goldberg, M. E., \& Robinson, D. L. (1982). Brain Mechanisms of Visual Attention. Scientific American, 246, 124-135. 